\title{
Diversity and Prevalence of Antibiotic Resistance Genes, Virulence Factors, and the Microbiome in Aquaculture in Southern China Revealed by Metagenomic Sequencing
}

\section{Haochang Su}

South China Sea Fisheries Research Institute

\section{Wujie Xu}

South China Sea Fisheries Research Institute

Xiaojuan Hu

South China Sea Fisheries Research Institute

$\mathrm{YuXu}$

south china sea fisheries

\section{Guoliang Wen}

South China Sea Fisheries Research Institute

Yucheng Cao ( $\nabla$ cyc_715@163.com )

South China Sea Fisheries Research Institute https://orcid.org/0000-0002-0995-1131

\section{Research}

Keywords: Antibiotic resistance genes, Virulence factors, Human pathogenic bacteria, Metagenomics, Human health, Aquaculture

Posted Date: November 4th, 2020

DOl: https://doi.org/10.21203/rs.3.rs-100086/v1

License: (c) (1) This work is licensed under a Creative Commons Attribution 4.0 International License. Read Full License 


\section{Abstract}

Background: Microbiota carrying multiple antibiotic resistance genes (ARGs) and virulence factors (VFs) are posing increasing risks to public health. Particularly the rapid spread of human pathogenic bacteria (HPB) with antibiotic resistance is recognized as a top health issue. The occurrence and abundance of ARGs in aquaculture have been investigated following metagenomic approaches. However, few studies have investigated the antibiotic resistome and VFs and their HPB hosts in aquaculture. Moreover, the relationships between ARGs and VFs and their microbiome in aquaculture are poorly understood.

Results: The profiles of the antibiotic resistome, VFs, and HPB in aquaculture in Southern China were investigated. In total, 492 subtypes of 24 ARGs types were detected. Multidrug ARGs were most predominant, followed by macrolide-lincosamide-streptogramin (MLS). Proteobacteria were the most predominant phylum carrying ARGs, followed by Firmicutes. Fifty-two HPB genera were detected. Firmicutes was the most abundant phylum, followed by Proteobacteria. Staphylococcus was the most abundant HPB genus. The samples contained 363 VFs, with Capsule being the most abundant. Seven HPB phyla, including 42 HPB genera, carried VFs, and the abundance of Bacillus was highest. The abundances of ARGs and VFs were highest in the sediment. However, the abundance of HPB was highest in shrimp guts and Staphylococcus was most abundant. Most ARGs were more prevalent on chromosomes than on plasmids. Source tracking analysis showed that the sediment was the greatest contributor to microbes carrying ARGs, VFs, and HPB in shrimp guts. Additionally, the water source contributed some of the HPB of shrimp guts.

Conclusions: This study provides in-depth profiles of the abundances, diversity, distribution, and prevalence of ARGs, VFs, and their hosts HPB in aquaculture for the first time. Sediment was the most direct and important contributor to the ARGs, VFs, and HPB in the shrimp guts. The prevalence of HPB in aquaculture, particularly the high abundance of Staphylococcus in shrimp guts, poses potential risks to human health and food safety. Aquaculture water sources should be monitored and protected. The findings of this study provide a better understanding of the dissemination and hosts of ARGs and VFs for improving aquaculture management and public health surveillance.

\section{Background}

Antibiotics resistance issues have attracted much public concern worldwide [1, 2]. According to the World Health Organization (WHO), antibiotic resistance is currently one of the greatest threats to global health, food security, and economic development, leading to longer hospital stays, higher medical costs, and increased mortality (https://www.who.int/en/news-room/fact-sheets/detail/antibiotic-resistance). An article published in Science reported that anthropogenic activities have considerably changed the movements of microorganisms and their genes by modifying selection pressures over the past 100 years. Accordingly, microbial biogeography is changing substantially [3]. Bacteria carrying multiple antibiotic resistance genes (ARGs) pose a higher potential risk to public health, particularly with the rapid spread of human pathogenic bacteria (HPB) with comprehensive antibiotic resistance [4]. 
Previous studies investigated the occurrence and concentrations of ARGs in aquaculture through different approaches, including the universal polymerase chain reaction (UPCR) $[5,6]$ and quantitative polymerase chain reaction (qPCR) $[7,8]$. However, the shortcomings of uPCR and qPCR have limited the study of the whole ARG profile, as they can only identify a limited number of ARGs. This limitation can be resolved by metagenomic sequencing. The changes in the microbiome and mobile genetic elements (MGEs) of the rearing water and fish gut exposed to antibiotics can be revealed by metagenomic analysis [9-11], along with the occurrence and abundance of ARGs and MGEs in aquaculture [12-15].

HPB carrying ARGs and virulence factors (VFs) pose a considerable risk to human health [4]. However, to the best of our best knowledge, only a few studies have investigated the profiles of the antibiotic resistome and VFs and their hosts in aquaculture. Moreover, the relationships between the ARGs and VFs and the microbiome carrying them in aquaculture remain unknown. This study aimed to profile the antibiotic resistome, VFs, and HPB, and their relationships, and analyze the microbiome carrying these genetic factors in aquaculture. The findings of this study offer a better understanding of the dissemination and hosts of ARGs and VFs and can aid in improving aquaculture management and the safety of aquatic products.

\section{Methods}

\section{Sample collection}

Duck and shrimp farms located in Guangdong, South China, were selected as study sites. The duck farm (Farm $1,113.505757^{\circ} \mathrm{E}, 22.614772^{\circ} \mathrm{N}$ ) rears over three thousand ducks in approximately ten ponds with an area of $6.8 \mathrm{ha}$; this is a popular duck rearing practice in this area. The shrimp farm (Farm 2, $113.516473^{\circ} \mathrm{E}, 22.673034^{\circ} \mathrm{N}$ ) covers an area of 7.1 ha with twelve rearing ponds. Litopenaeus vannamei was the predominant reared organism, with a stocking density of 900,000 shrimps per hectare in each pond and was polycultured with one-hundred grass carp (Ctenopharyngodon idellus). Samples were collected from three ponds on each farm. Approximately 100 adult shrimps were aseptically collected from each shrimp pond in sterile plastic bags, while approximately $500 \mathrm{~g}$ of duck feces samples were aseptically collected from each duck pond. The rivers near the two farms that served as water sources were also sampled. The sampling methods for the water and sediment in the water sources and rearing ponds were detailed in our previous study [16]. Samples from the three rearing ponds for each farm were collected in triplicates. All collected samples were stored in a freezer box and transported to the laboratory for treatment within $24 \mathrm{~h}$.

\section{DNA extraction}

Approximately $0.5 \mathrm{~L}$ of each water sample was filtered through a sterile membrane filter with a pore size of $0.2 \mu \mathrm{m}$ (Merck Millipore, Ireland), which was, then, stored aseptically at $-80^{\circ} \mathrm{C}$ for DNA extraction. The sediment and duck feces samples were lyophilized, ground, and sieved through an 80-mesh screen. The 
DNAs in the water, sediment, and duck feces samples were extracted using a PowerSoil DNA Isolation Kit (Mobio, USA) following the protocol by the manufacturer and our previous study [16]. The shrimp gut samples were separated aseptically and homogenized using a shaking machine. The DNA of the shrimp gut samples was extracted using a HiPure Stool DNA Kit B (Magen, China) following the protocol provided by the manufacturer, as described in our previous study [16]. Three DNA replicates were taken for each sample for the subsequent metagenomic sequencing.

\section{Metagenomic sequencing and data analysis}

The DNA samples were sent to Mingke Biotechnology (Hangzhou) Co., Ltd., China for Illumina shotgun high-throughput sequencing using the $150 \mathrm{PE}$ sequencing strategy (paired-end sequencing, 150-bp reads). Approximately $10 \mathrm{~GB}$ of raw data were generated for each sample. The sequences obtained were saved in the National Center for Biotechnology Information (NCBI) database (SRA accession:

PRJNA648777). Raw sequences, including those smaller than $50 \mathrm{bp}$, with degenerate bases (N's), and those with an average quality score below 20 were filtered using Trimmomatic [36]. The filtered clean reads were assembled into contigs using Megahit [37]. Genes prediction was, then, conducted by applying Prodigal [38], and the gene files of the samples were obtained. Nonredundant gene sets with less than $90 \%$ overlap and less than $95 \%$ shared sequence were constructed from the gene files with CDHIT [39]. The clean reads of each sample were, then, mapped to the clean nonredundant gene sets using salmon [40], and the abundance transcripts per million reads (TPM) of these nonredundant gene sets for each sample were obtained. These genes were also blasted against the NR database in NCBI to obtain the putative taxon assignments for each sample using diamond [41].

\section{ARGs and bacterial ARG taxon annotations}

The annotations of the patterns and bacterial taxa of ARGs were obtained from Hu et al. (2020) [42]. Briefly, the genes were blasted against the Structured Antibiotic Resistance Genes database (SARG version 2.0) [43] with an E-value of $\leq 10^{-7}$ to obtain the putative sequences of ARGs. Based on the TPM abundance, the abundance of ARG types and subtypes for each sample (TPM) were obtained using custom Perl scripts [42]. The ARG sequences were blasted against the NR database in NCBI to obtain the bacterial taxon of ARGs for each sample using diamond [41]. The abundance (TPM) of the bacterial taxa for the ARG types and subtypes in each sample was obtained with custom Perl scripts.

\section{Identification of HPB and VFs}

HPB were identified by blasting against the HPB 16S, which are publicly available from the NCBI GenBank (http://www.ncbi.nlm.nih.gov/), following the study of Fang et al. [44]. Genes for each sample were blasted against the virulence factor database (http://www.mgc.ac.cn/VFs/) to identify VFs following the study of Chen et al. (2012) [28]. 


\section{Detection of ARGs in chromosomes or plasmids and source tracking}

The presence of ARGs in chromosomes or plasmids was determined using BLAST+ blastx against $1,044,458$ complete plasmid sequences from the NCBI RefSeq database (updated in October 2019) following Fresia et al. [29]. Hits with query coverage of over $90 \%$ amino and acid identification of over $70 \%$ were retained. Taxonomic classification was determined from the description header of both plasmids and chromosomes. Source tracking analysis of the HPB and microbiota carrying ARGs or VFs was conducted using Source Tracker (V0.9.5) in R (V3.4.4) following Knights et al. [45].

\section{Results}

\section{Prevalence and abundance of ARGs and microbiota carrying ARGs}

Twenty-four types of ARG were detected in the samples (Fig. 1a). Multidrug ARGs were the most predominant, with an average abundance of $4,710 \mathrm{ppm}$, followed by macrolide-lincosamidestreptogramin (MLS; 4,570 ppm) and vancomycin (4,150 ppm) ARGs. A total of 492 subtypes of ARGs were identified. The macB subtype of MLS ARGs was the most abundant (ranging from 1,060 to 6,420 ppm, with an average of $3,590 \mathrm{ppm})$, followed by $b c r A$, a subtype of bacitracin ARGs (2,380 ppm), and vanS, a subtype of vancomycin ARGs (1,540 ppm).

The abundances of ARGs in each sample ranged from 5,800 to $39,500 \mathrm{ppm}$ (Fig. 1a). Sediment sample S1 contained the most ARGs, with an average abundance of $38,800 \mathrm{ppm}$, followed by the duck feces sample (33,000 ppm), and sediment sample S2 (32,400 ppm). The shrimp gut sample (SI) had the lowest abundance of ARGs (5,850 ppm).

The most predominant phylum carrying ARGs were the Proteobacteria (Fig. 1b), with abundances ranging from 355 to 18,300 ppm, with an average of 7,720 ppm, followed by Firmicutes (3,260 ppm), Chloroflexi (2,510 ppm), Actinobacteria (1,790 ppm), Bacteroidetes (1,760 ppm), Cyanobacteria (1,200 ppm), Planctomycetes (441 ppm), Verrucomicrobia (282 ppm), and Acidobacteria (173 ppm).

Chloroflexi_norank was found to be the most abundant genus carrying ARGs, with an average abundance of 1,190 ppm, followed by Bacillus (757 ppm), Limnohabitans (710 ppm), Lactococcus (701 ppm), and Anaeromyxobacter (409 ppm). The results of circos analysis showed that Proteobacteria, Cyanobacteria, Firmicutes, Actinobacteria, Chloroflexi, Bacteroidetes, Planctomycetes, Verrucomicrobia, Nitrospirae, and Acidobacteria contributed $94.49 \% \sim 99.85 \%$ of the abundances of the most predominent ARGs, e.g. macB, $b c r A$, vanS, vanR, ompR, truncated-ArIR and ABC_transporter in aquaculture (Fig. 2a). Among these phyla, the most predominant phylum contributing for ARGs were the Proteobacteria, with the contribution of $27.38 \% \sim 59.49 \%$ of the abundance of the most predominent ARGs, and with contributing $40.18 \%$ of the abundance of $m a c B$, the most abundant ARG subtypes in aquaculture. For genera, Anaeromyxobacter, 
Planktothricoides, Lactococcus, Bacillus, Limnohabitans, Caldilinea, Planktothrix, Synechococcus, Cyanobium, and Desulfuromonas also contributed $26.66 \%$ 51.63\% of the abundance of the most predominent ARGs, e.g. macB, bcrA, vanS, vanR and ABC_transporter in aquaculture (Fig. 2b).

Lactococcus contributed $8.80 \%, 8.63 \%$ and $3.36 \%$ of the abundances of $m a c B, b c r A$, and vanS, the three most abundant ARG subtypes, respectively. Besides, Lactococcus contributed $14.61 \%$ and $11.74 \%$ of the abundances of cystathionine-patB and ABC_transporter, respectively.

Sediment sample $\mathrm{S} 1$ contained the most microbes carrying ARGs (Fig. 1b), with an average abundance of 38,800 ppm, followed by duck feces sample DF (33,000 ppm), sediment sample $S 2$ (32,400 ppm), water source sample WS1 (28,300 ppm), pond water sample PW1 (18,900 ppm), pond water sample PW2 $(16,600 \mathrm{ppm})$, and water source sample WS2 (16,100 ppm). Shrimp gut sample SI contained the least microbiota carrying ARGs, with an abundance of $5850 \mathrm{ppm}$.

\section{Prevalence of HPB}

HPB were prevalent in shrimp aquaculture. Fifty-two genera belonging to eight phyla were detected (Fig. 3). Firmicutes was the most abundant, with an average abundance of $11,900 \mathrm{ppm}$ among the samples, followed by Proteobacteria (1,490 ppm) and Actinobacteria (526 ppm). Staphylococcus, a member of the Firmicutes phylum, was the most abundant HPB genus among the samples, with an average abundance of 5,820 ppm, followed by Bacillus (4,260 ppm), Clostridium (965 ppm), and Streptococcus (813 ppm).

The total abundances of HPB in each sample ranged from 963 to 29,300 ppm. The shrimp gut samples had the highest total abundance of HPB, with an average of 29,100 ppm (most of which were Staphylococcus; 16,000 ppm), followed by sediment sample S2 (25,300 ppm), S1 (19,400 ppm), and the feces sample (9,750 ppm). The total abundances of HPB in pond water samples PW1 and PW2 were 1,050 and 1,090 ppm, respectively. The water source of Farm 2, WS2, had the lowest total abundance of HPB (1,000 ppm). A high abundance of HPB was identified in the water source of Farm 1, WS1 (1,470 $\mathrm{ppm})$, which was even higher than those of the pond water samples.

\section{Prevalence of VFs and microbiota carrying VFs}

A total of 363 VFs were identified in the samples (Fig. 4a), of which VF Capsule was the most abundant, ranging from 1,350 to $7,810 \mathrm{ppm}$, with an average abundance of $4,890 \mathrm{ppm}$, followed by lipopolysaccharide (LPS, 4,610 ppm), Flagella (2,810 ppm), and Polar flagella (2,730 ppm). The abundances of VFs ranged from 13,500 to 94,600 ppm in each sample, and sediment sample $S 1$ contained the most VFs, with an average abundance of $92,000 \mathrm{ppm}$, followed by sediment sample $\mathrm{S} 2$ $(80,700 \mathrm{ppm})$, water source sample WS1 (79,500 ppm), and the duck feces sample (78,700 ppm). Shrimp gut sample SI contained the least VFs, with an average abundance of $13,600 \mathrm{ppm}$. 
Seven HPB phyla containing 42 HPB genera carrying VFs were identified. Firmicutes was the most predominent phylum, with an average abundance of 362 ppm, followed by Proteobacteria. For HPB genera, Bacillus was the most abundant, with an average abundance of $253.5 \mathrm{ppm}$, followed by Clostridium (22.26 ppm). Circos analysis showed that Firmicutes, Proteobacteria, Actinobacteria, Bacteroidetes, Spirochaetes, Fusobacteria and Chlamydiae contributed $99.99 \%$ 100\% of the abundances of the most predominent VFs, e.g. Capsule, LPS, Flagella, Capsule-I, HitABC, and Polar-flagella, etc in aquaculture (Fig. 5a). Among these HPB phyla, the largest contributor for VFs was Firmicutes, with the contribution of $58.37 \% \sim 89.41 \%$ of the abundance of the most predominent VFs, and with contributing $78.73 \%$ of the abundance of Capsule, the most abundant VF in aquaculture. For HPB genera, Bacillus, Mycobacterium, Pseudomonas, Streptococcus, Bordetella, Clostridium, Aeromonas, Actinomadura, Bacteroides and Vibrio contributed $92.67 \%$ 99.61\% of the abundance of the most predominent VFs, e.g. Capsule, LPS, Flagella, Colibactin, HitABC, LOS, and Polar flagella (Fig. 5b). Bacillus contributed $52.94 \% \sim 86.14 \%$ of the abundance of the most predominent VFs, and $72.53 \%, 52.94 \%$ and $78.61 \%$ of the abundances of Capsule, LPS and Flagella, the three most abundant VFs, respectively.

Sediment sample S2 contained the most abundant HPB carrying VFs (Fig. 4b), with an average abundance of 1,005 ppm, followed by S1 (746 ppm) and the duck feces sample ( $617 \mathrm{ppm})$. The average abundance of HPB carrying VFs in the shrimp gut samples was 530 ppm, with Bacillus being the most predominent HPB genera, with the proportion of $95.07 \%$, followed by Streptococcus (1.99\%) and Staphylococcus (0.84\%).

\section{Occurrence of ARGs on the chromosomes and plasmids}

Twenty types of ARG were identified on both the chromosomes and plasmids; most of them were found on the chromosomes, with an average abundance of 8,840 ppm (62.3\%) (Fig. 6a). All ARG types were more prevalent on the chromosomes than on the plasmids, with proportions ranging from $53.4 \%$ (trimethoprim ARGs) to $84.2 \%$ (kasugamycin ARGs), excluding aminoglycoside ARGs, whose proportion on the plasmids was $56.4 \%$. MLS ARGs were the most abundant, with a value of $3,304 \mathrm{ppm}(23.3 \%)$, followed by tetracycline $(2,700 \mathrm{ppm}, 19.1 \%)$ and multidrug $(2,340 \mathrm{ppm}, 16.5 \%)$ ARGs.

Among the different samples, the duck feces samples contained the most amount of ARGs on the chromosomes and plasmids (Fig. 6b), with an abundance of 27,000 ppm, followed by the sediment $(15,500 \mathrm{ppm})$ and water $(11,100 \mathrm{ppm})$ samples. The shrimp gut samples contained the lowest amount of $\operatorname{ARGs}(3,190 \mathrm{ppm})$.

\section{Source tracking results}

Figure 7a shows the results of the source tracking of microbiota in the sediment. Unknown microbiota accounted for the largest proportion of the microbiome and contained $59.6 \%, 66.2 \%$, and $99.9 \%$ of the ARGs, VFs, and HPB groups, respectively; the second largest proportion of the microbiome was found in 
pond water, followed by the water source samples. Duck feces contributed to $1.06 \%-1.51 \%$ of microbiota in the sediment.

The source of the microbiota of shrimp guts is presented in Fig. 7b. Sediment was the largest contributor, accounting for $62.2 \%, 75.1 \%$, and $89.5 \%$ of the ARGs, VFs, and HPB groups, respectively, followed by the unknown source and water sources.

\section{Discussion}

Most previous studies on the occurrence and concentrations of ARGs in aquaculture were conducted following uPCR and qPCR approaches [7, 8, 16-21]. However, owing to the limits of uPCR and qPCR, the full profiles of ARGs could not be described. The metagenomic analysis may be used to overcome these limitations. In this study, 492 subtypes of 24 types of ARGs were detected in the aquaculture system. macB was the most abundant ARG subtype, conferring resistance to macrolides, which could be attributed to the high amount of residual macrolide antibiotics in the feed [22, 23]. The vanS subtype of ARGs, conferring resistance to vancomycin, was the third-most abundant in the aquaculture system. The use of vancomycin in livestock and aquaculture was banned by the Ministry of Agriculture and Rural Affairs, People's Republic of China in 2001. These results suggest that ARGs would persist for over a decade without the selective pressure of antibiotics. Through metagenomic analysis, Chen et al. found that the total abundance of ARGs in the sediment of bullfrog farms ranged from 8.6 to $111.2 \mathrm{ppm}$ [12]. Higher prevalence and abundance of ARGs were detected in this study. The total abundances of ARGs in each sample ranged from 5800 to $39,500 \mathrm{ppm}$, and the sediment sample contained the most ARGs, with an average abundance of $38,800 \mathrm{ppm}$.

Plasmids, as an important MGE, play a key role in the dissemination of ARGs in the environment [24]. Che et al. [25] found that the ARGs in the MGEs accounted for $55 \%$ of the total ARGs in samples from a wastewater treatment plant, whereas those on the chromosome accounted for $29 \%$. However, a higher prevalence of ARGs was observed on the chromosome in this study, accounting for an average of $62.3 \%$. Che et al. detected higher proportions of ARGs in the MGEs most likely because mobile genes flow and communicate most frequently in wastewater $[24,26]$.

Analyzing the microbiota carrying ARGs and/or VFs aided in understanding the occurrence, source, and distribution of ARGs and/or VFs in aquaculture systems. Zeng et al. reported that the abundances of Proteobacteria, Bacteroidetes, Actinobacteria, and Verrucomicrobia increased in the gut of catfish exposed to standard therapeutic 10-d florfenicol treatment and inferred that these bacteria either harbor florfenicol resistance genes (FRGs) or is characterized by beneficial mutations that lead to their increased abundance [10]. In this study, Proteobacteria was the most predominant microbiota carrying ARGs in the aquaculture system, followed by Firmicutes, Chloroflexi, Actinobacteria, Bacteroidetes, Cyanobacteria, Planctomycetes, and Verrucomicrobia. The producer hypothesis states that the ARGs in other bacteria could have originated from Actinobacteria that produce antibiotics by ancient horizontal gene transfer 
(HGT) [27]. From the results of this study and the previous study, it could be inferred that Proteobacteria is currently the main contributor to the dissemination of ARGs, rather than Actinobacteria.

VFs provide beneficial mechanisms and help pathogens to establish infections, cause diseases, and survive in disadvantageous environments [28]. Fresia et al. reported that the abundance and diversity of VFs in sewage samples were higher than those in beach samples, and VFs involving bacterial motility, cell adherence, iron uptake, and secretion were predominant in the sewage samples. They concluded that urban waters served as a reservoir and medium for VFs responsible for clinically relevant bacteria and their transport [29]. Unlike the results of Fresia et al. [29], VFs such as Capsule with the antiphagocytosis function, LPS, with the endotoxin function, and Flagella, with the invasion function, were predominant in our study. HPB carrying VFs with diverse functions were observed in each aquaculture sample, even in the reared shrimp, posing high risks to human health and food safety.

HPB can easily capture multiple ARGs and form multidrug-resistant (MDR) bacteria and even Superbugs [30-32]. Additionally, HPB harbor VFs with diverse functions [28]. Therefore, humans are readily infected by HPB with MDR genes and pathogenicity via contact or the consumption of raw vegetables [33]. Che et al. [25] and Fresia et al. [29] observed high HPB abundance in wastewater samples, which is likely because the hospital and domestic wastewater containing samples of the human gut microbiome converge and are treated in the sewage system, from which HPB are discharged into the environment through the effluent pipes [26]. In this study, 52 genera of HPB were identified in the shrimp gut samples, which had VF and HPB abundances of 13,600 and 29,100 ppm, respectively. Among the HPB phyla, Firmicutes contributed $58.37 \% \sim 89.41 \%$ of the abundance of the most predominent VFs. For HPB genera, Staphylococcus was the most abundant HPB in the shrimp gut, with an abundance of $16,000 \mathrm{ppm}$. Other HPB present included Aeromonas, Bacillus, Clostridium, Streptococcus, Salmonella, Serratia, and Mycobacterium. Staphylococcus is a highly pathogenic bacteria and Superbug that can cause severe infections even lethality, particularly the common methicillin-resistant Staphylococcus aureus (MRSA) with MDR genes [34]. Overall, the findings of our study and those of previous studies indicate that the presence of HPB in aquaculture, particularly in reared shrimp, poses severe risks to human health and food safety, and the source of HPB should be investigated and traced to improve public health surveillance.

The source tracking results indicate that unknown microbiota contained most of the ARGs, VFs, and HPB in the sediment, Therefore, more sources should be considered in the analysis besides the source water, pond water, and duck feces samples in future studies. Sediment was found to contribute the most ARGs, VFs, and HPB to the shrimp gut samples, which was consistent with the findings of our previous study [35]; this demonstrates that the sediment is the most direct and important medium in the dissemination of ARGs in aquaculture. Meanwhile, water source contributed $4.70 \%$ of the VFs and $7.42 \%$ of HPB in the shrimp gut samples, suggesting that the water source used in aquaculture should be monitored and protected to avoid the risks posed by VFs and HPB to human health and food safety.

\section{Conclusions}


This study provides in-depth profiles of the prevalence and distribution of ARGs, VFs, and HPB in aquaculture. High abundances and diversity of ARGs, VFs, and HPB were observed in duck feces, water source, pond water, sediment, and shrimp gut samples. Proteobacteria were the most predominant microbiota carrying ARGs, and the prevalence of ARGs in the microbial chromosomes was higher than that in the plasmids. Capsule was the most abundant VF and Firmicutes was the most abundant HPB, followed by Proteobacteria. The sediment was the most direct and important medium that contributed to the concentrations of ARGs, VFs, and HPB in the shrimp guts. The presence of HPB in aquaculture systems, particularly the high abundance of Staphylococcus in shrimp guts, poses a severe risk to human health and food safety. The water source of aquaculture systems should be supervised and preserved. The findings of this study provide a better understanding of the dissemination and hosts of ARGs and VFs and can aid in improving aquaculture management and public health surveillance.

\section{Abbreviations}

ARGs - Antibiotic-resistance genes

VFs - Virulence factors

LPS - Lipopolysaccharide

HPB - Human pathogenic bacteria

MGEs - Mobile genetic elements

MLS - Macrolide-lincosamide-streptogramin

WHO - World Health Organization

UPCR - Universal polymerase chain reaction

qPCR - Quantitative polymerase chain reaction

FRGs - Florfenicol resistance genes

HGT - Horizontal gene transfer

MDR - Multidrug-resistant

MRSA - Methicillin-resistant Staphylococcus aureus

TPM - Transcripts per million reads

\section{Declarations}

Ethics approval and consent to participate 


\section{Consent for publication}

Not applicable.

\section{Availability of data and material}

The datasets generated from Illumina sequencing were saved into the National Center for Biotechnology Information database under the following accession number SRA accession PRJNA648777.

\section{Competing interests}

The authors declare that they have no competing interests.

\section{Funding}

This study was funded by the National Key R\&D Program of China (2019YFD0900402), the Central Public-interest Scientific Institution Basal Research Fund, CAFS (NO. 2020TD54), the Science and Technology Program of Guangzhou, China (202002030496), the Central Public-interest Scientific Institution Basal Research Fund, South China Sea Fisheries Research Institute, CAFS (NO. 2020XK02), the Natural Science Foundation of Guangdong Province (2019A1515011618), the Natural Science Foundation of China (NSFC41501529), the China Agriculture Research System (CARS-48).

\section{Authors' contributions}

HS and $Y C$ designed this research. $W X, X H, G W$ and $Y X$ collected samples and conducted experiments. $\mathrm{HS}$ and $\mathrm{YC}$ analyzed the data and wrote the manuscript. All authors revised and approved the final manuscript.

\section{Acknowledgments}

The authors thank Hua Chen, the chief technology officer from Mingke Biotechnology (Hangzhou) Co., Ltd., China, for his help of professional bioinformatics analysis.

\section{Authors' information}


${ }^{1}$ Key Laboratory of South China Sea Fishery Resources Exploitation \& Utilization, Ministry of Agriculture and Rural Affairs, P.R.China; South China Sea Fisheries Research Institute, Chinese Academy of Fishery Sciences, Guangzhou 510300, China

${ }^{2}$ Guangdong Provincial Key Laboratory of Fishery Ecology and Environment

${ }^{3}$ Shenzhen Base South China Sea Fisheries Research Institute, Chinese Academy of Fishery Sciences, Shenzhen, 518121, China

\section{References}

1. Pruden A, Pei RT, Storteboom H, Carlson KH. Antibiotic resistance genes as emerging contaminants: Studies in northern Colorado. Environ. Sci. Technol. 2006;40(23):7445-50.

2. Pruden A, Larsson DGJ, Amézquita A, Collignon P, Brandt KK, Graham DW, Lazorchak JM, Suzuki S, Silley P, Snape JR, Topp E, Zhang T, Zhu YG. Management Options for Reducing the Release of Antibiotics and Antibiotic Resistance Genes to the Environment. Environ. Health Perspect. 2013;121(8):878-85.

3. Zhu YG, Gillings M, Simonet P, Stekel D, Banwart S, Penuelas J. Microbial mass movements. Science. 2017;357(6356):1099-1100.

4. Robinson TP, Bu DP, Carrique-Mas J, Fevre EM, Gilbert M, Grace D, Hay SI, Jiwakanon J, Kakkar M, Kariuki S, Laxminarayan R, Lubroth J, Magnusson U, Ngoc PT, Van Boeckel TP, Woolhouse MEJ. Antibiotic resistance is the quintessential One Health issue. Trans. R. Soc. Trop. Med. Hyg. 2016;110(7):377-80.

5. Stalin N, Srinivasan P. Molecular characterization of antibiotic resistant Vibrio harveyi isolated from shrimp aquaculture environment in the south east coast of India. Microb. Pathog. 2016;97:110-8.

6. Singh B, Tyagi A, Thammegowda NKB, Ansal MD. Prevalence and antimicrobial resistance of vibrios of human health significance in inland saline aquaculture areas. Aquacult. Res. 2018;49(6):2166-74.

7. Yuan JL, Ni M, Liu M, Zheng Y, Gu ZM. Occurrence of antibiotics and antibiotic resistance genes in a typical estuary aquaculture region of Hangzhou Bay, China. Mar. Pollut. Bull. 2019;138:376-84.

8. Su HC, Hu XJ, Xu Y, Xu WJ, Huang XS, Wen GL, Yang K, Li ZJ, Cao YC. Persistence and spatial variation of antibiotic resistance genes and bacterial populations change in reared shrimp in South China. Environ. Int. 2018;119:327-33.

9. Saenz JS, Marques TV, Barone RSC, Cyrino JEP, Kublik S, Nesme J, Schloter M, Rath S, Vestergaard G. Oral administration of antibiotics increased the potential mobility of bacterial resistance genes in the gut of the fish Piaractus mesopotamicus. Microbiome. 2019;7:24-37.

10. Zeng QF, Liao C, Terhune J, Wang LX. Impacts of florfenicol on the microbiota landscape and resistome as revealed by metagenomic analysis. Microbiome 2019;7(1):155-67.

11. Almeida AR, Alves M, Domingues I, Henriques I. The impact of antibiotic exposure in water and zebrafish gut microbiomes: A 16S rRNA gene-based metagenomic analysis. Ecotoxicol. Environ. Saf. 
2019;186:109771.

12. Chen B, Lin L, Fang L, Yang Y, Chen E, Yuan K, Zou S, Wang X, Luan T. Complex pollution of antibiotic resistance genes due to beta-lactam and aminoglycoside use in aquaculture farming. Water Res. 2018;134:200-8.

13. Zhao YT, Zhang XX, Zhao ZH, Duan CL, Chen HG, Wang MM, Ren HQ, Yin Y, Ye L. Metagenomic analysis revealed the prevalence of antibiotic resistance genes in the gut and living environment of freshwater shrimp. J. Hazard. Mater. 2018;350:10-8.

14. Fang H, Huang KL, Yu JN, Ding CC, Wang ZF, Zhao C, Yuan HZ, Wang Z, Wang S, Hu JL, Cui YB. Metagenomic analysis of bacterial communities and antibiotic resistance genes in the Eriocheir sinensis freshwater aquaculture environment. Chemosphere 2019;224:202-11.

15. Liu KX, Han JM, Li SR, Liu LT, Lin WT, Luo JF. Insight into the diversity of antibiotic resistance genes in the intestinal bacteria of shrimp Penaeus vannamei by culture-dependent and independent approaches. Ecotoxicol. Environ. Saf. 2019;172:451-9.

16. Su HC, Liu S, Hu XJ, Xu XR, Xu WJ, Xu Y, Li ZJ, Wen GL, Liu YS, Cao YC. Occurrence and temporal variation of antibiotic resistance genes (ARGs) in shrimp aquaculture: ARGs dissemination from farming source to reared organisms. Sci. Total Environ. 2017;607:357-66.

17. Su HC, Ying GG, Tao R, Zhang RQ, Fogarty LR, Kolpin DW. Occurrence of antibiotic resistance and characterization of resistance genes and integrons in Enterobacteriaceae isolated from integrated fish farms in south China. J. Environ. Monit. 2011;13(11):3229-36.

18. Huang L, Xu YB, Xu JX, Ling JY, Chen JL, Zhou JL, Zheng L, Du QP. Antibiotic resistance genes (ARGs) in duck and fish production ponds with integrated or non-integrated mode. Chemosphere 2017;168:1107-14.

19. Xiong WG, Sun YX, Zhang T, Ding XY, Li YF, Wang MZ, Zeng ZL. Antibiotics, Antibiotic Resistance Genes, and Bacterial Community Composition in Fresh Water Aquaculture Environment in China. Microb. Ecol. 2015;70(2):425-32.

20. Muziasari WI, Managaki S, Parnanen K, Karkman A, Lyra C, Tamminen M, Suzuki S, Virta M. Sulphonamide and trimethoprim resistance genes persist in sediments at Baltic Sea aquaculture farms but are not detected in the surrounding environment. PLoS One. 2014;9(3):e92702.

21. Muziasari WI, Pärnänen K, Johnson TA, Lyra C, Karkman A, Stedtfeld RD, Tamminen M, Tiedje JM, Virta M, Smalla K. Aquaculture changes the profile of antibiotic resistance and mobile genetic element associated genes in Baltic Sea sediments. FEMS Microbiol. Ecol. 2016;92(4):fiw052.

22. Chen H, Liu S, Xu XR, Diao ZH, Sun KF, Hao QW, Liu SS, Ying GG. Tissue distribution, bioaccumulation characteristics and health risk of antibiotics in cultured fish from a typical aquaculture area. J. Hazard. Mater. 2018;343:140-8.

23. Chen H, Liu S, Xu XR, Liu SS, Zhou GJ, Sun KY, Zhao JL, Ying GG. Antibiotics in typical marine aquaculture farms surrounding Hailing Island, South China: Occurrence, bioaccumulation and human dietary exposure. Mar. Pollut. Bull. 2015;90(1-2):181-7. 
24. Stokes HW, Gillings MR. Gene flow, mobile genetic elements and the recruitment of antibiotic resistance genes into Gram-negative pathogens. FEMS Microbiol. Rev. 2011;35(5):790-819.

25. Che Y, Xia Y, Liu L, Li AD, Yang Y, Zhang T. Mobile antibiotic resistome in wastewater treatment plants revealed by Nanopore metagenomic sequencing. Microbiome 2019;7:44-55.

26. Newton RJ, McLellan SL, Dila DK, Vineis JH, Morrison HG, Eren AM, Sogin ML. Sewage Reflects the Microbiomes of Human Populations. mBio. 2015;6(2):e02574-14.

27. Jiang XL, Ellabaan MMH, Charusanti P, Munck C, Blin K, Tong YJ, Weber T, Sommer MOA, Lee SY. Dissemination of antibiotic resistance genes from antibiotic producers to pathogens. Nat. Commun. 2017;8:15784.

28. Chen L, Xiong Z, Sun L, Yang J, Jin Q. VFDB 2012 update: toward the genetic diversity and molecular evolution of bacterial virulence factors. Nucleic Acids Res. 2012;40:641-5.

29. Fresia P, Antelo V, Salazar C, Gimenez M, D'Alessandro B, Afshinnekoo E, Mason C, Gonnet GH, Iraola G. Urban metagenomics uncover antibiotic resistance reservoirs in coastal beach and sewage waters. Microbiome. 2019;7:35.

30. Fischbach MA, Walsh CT. Antibiotics for Emerging Pathogens. Science. 2009;325(5944):1089-93.

31. Forsberg KJ, Reyes A, Wang B, Selleck EM, Sommer MOA, Dantas G. The Shared Antibiotic Resistome of Soil Bacteria and Human Pathogens. Science. 2012;337(6098):1107-11.

32. Negreanu Y, Pasternak Z, Jurkevitch E, Cytryn E. Impact of Treated Wastewater Irrigation on Antibiotic Resistance in Agricultural Soils. Environ. Sci. Technol. 2012;46(9):4800-8.

33. Wheeler C, Vogt TM, Armstrong GL, Vaughan G, Weltman A, Nainan OV, Dato VM, Xia G, Waller K, Amon JJ. An outbreak of hepatitis a associated with green onions. N. Engl. J. Med. 2005;353(9):8907.

34. Cheng CWR, Ong CH, Chan DSG. Impact of BD Kiestra InoqulA streaking patterns on colony isolation and turnaround time of methicillin-resistant Staphylococcus aureus and carbapenem-resistant Enterobacterale surveillance samples. Clin. Microbiol. Infect. 2020;26(9):1201-6.

35. Su HC, Hu XJ, Wang LL, Xu WJ, Xu Y, Wen GL, Li ZJ, Cao YC, Su H, Hu X, Wang L, Xu W, Xu Y, Wen G, $\mathrm{Li} Z$, Cao Y. Contamination of antibiotic resistance genes (ARGs) in a typical marine aquaculture farm: source tracking of ARGs in reared aquatic organisms. J. Environ. Sci. Health Part B. 2020;55(3):220-9.

36. Bolger AM, Lohse M, Usadel B. Trimmomatic: a flexible trimmer for Illumina sequence data. Bioinformatics. 2014;30(15):2114-20.

37. Li D, Luo R, Liu C-M, Leung C-M, Ting H-F, Sadakane K, Yamashita H, Lam TW. MEGAHIT v1.0: A fast and scalable metagenome assembler driven by advanced methodologies and community practices. Methods. 2016;102:3-11.

38. Hyatt D, LoCascio PF, Hauser LJ, Uberbacher EC. Gene and translation initiation site prediction in metagenomic sequences. Bioinformatics. 2012;28(17):2223-30. 
39. Li W, Godzik A. Cd-hit: a fast program for clustering and comparing large sets of protein or nucleotide sequences. Bioinformatics. 2006;22(13):1658-9.

40. Patro R, Duggal G, Love MI, Irizarry RA, Kingsford C. Salmon provides fast and bias-aware quantification of transcript expression. Nat. Methods. 2017;14(4):417-26.

41. Buchfink B, Xie C, Huson DH. Fast and sensitive protein alignment using DIAMOND. Nat. Methods. 2015;12(1):59-60.

42. Hu T, Dai QL, Chen H, Zhang Z, Dai Q, Gu XD, Yang XY, Yang ZS, Zhu LF. Geographic pattern of antibiotic resistance genes in the metagenomes of the giant panda. Microb. Biotechnol. 2020;0(0):112.

43. Yin XL, Jiang XT, Chai BL, Li LG, Yang Y, Cole JR, Tiedje JM, Zhang T. ARGs-OAP v2.0 with an expanded SARG database and Hidden Markov Models for enhancement characterization and quantification of antibiotic resistance genes in environmental metagenomes. Bioinformatics. 2018;34(13):2263-70.

44. Fang $\mathrm{H}$, Wang $\mathrm{H}$, Cai $\mathrm{L}$, Yu Y. Prevalence of antibiotic resistance genes and bacterial pathogens in long-term manured greenhouse soils as revealed by metagenomic survey. Environ. Sci. Technol. 2014;49(2):1095-104.

45. Knights D, Kuczynski J, Charlson ES, Zaneveld J, Mozer MC, Collman RG, Bushman FD, Knight R, Kelley ST. Bayesian community-wide culture-independent microbial source tracking. Nat. Methods. 2011;8(9):761-5.

\section{Figures}

a

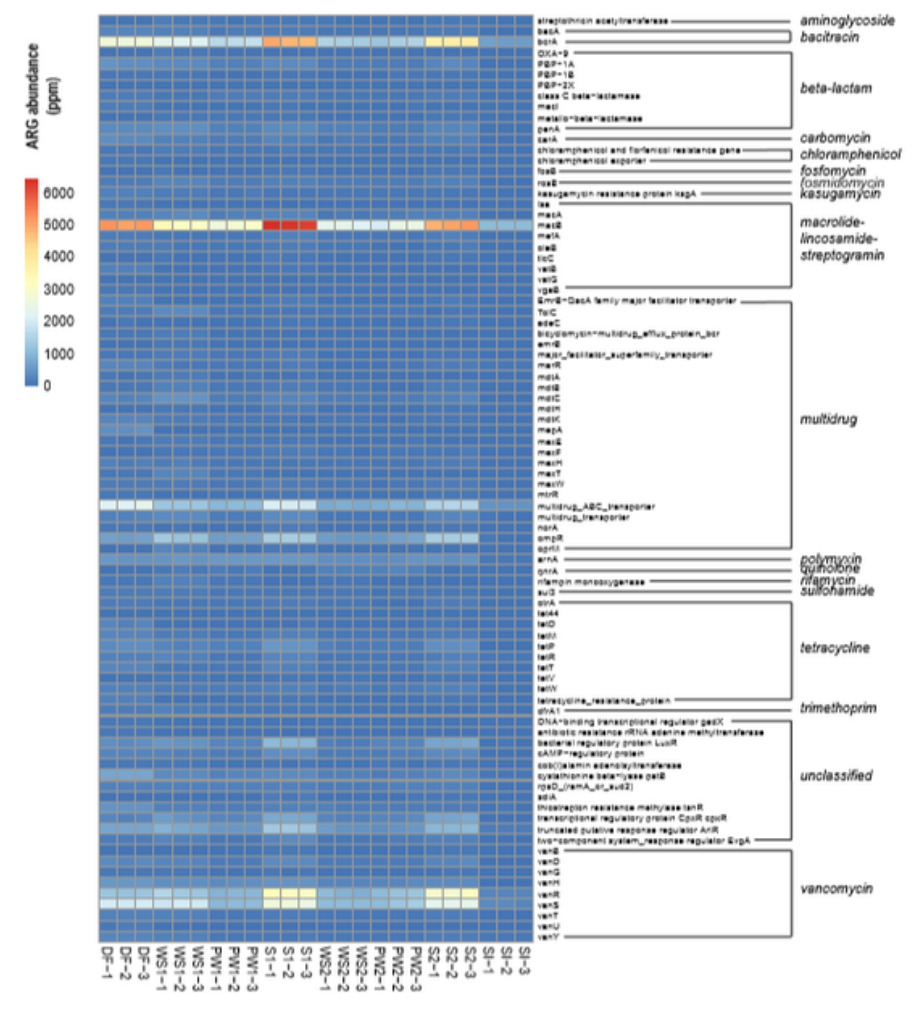

b

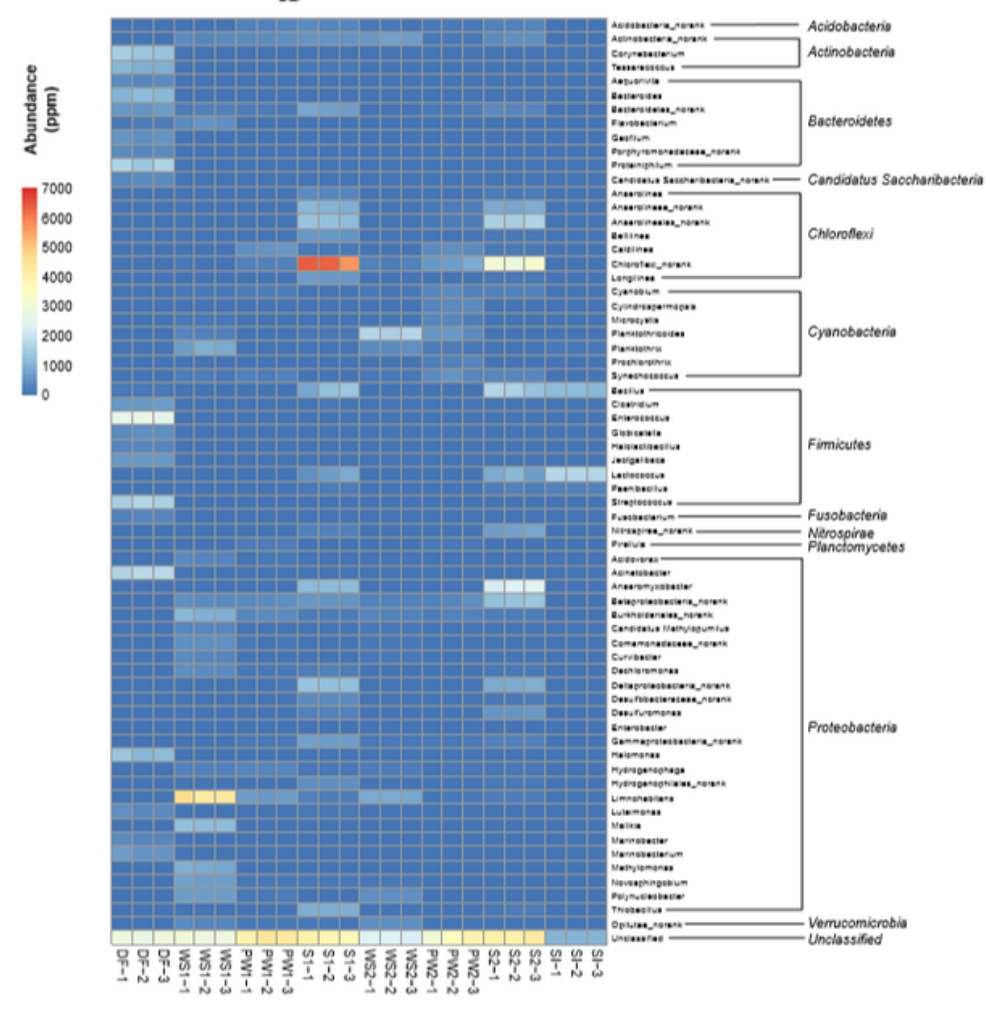


Figure 1

Abundances of antibiotic resistance genes (ARGs) and microbiota carrying ARGs for the samples in aquaculture. a Heatmap showing the abundances of ARG types and subtypes. $b$ Heatmap showing the abundances of microbiota carrying ARGs for phyla and genera.
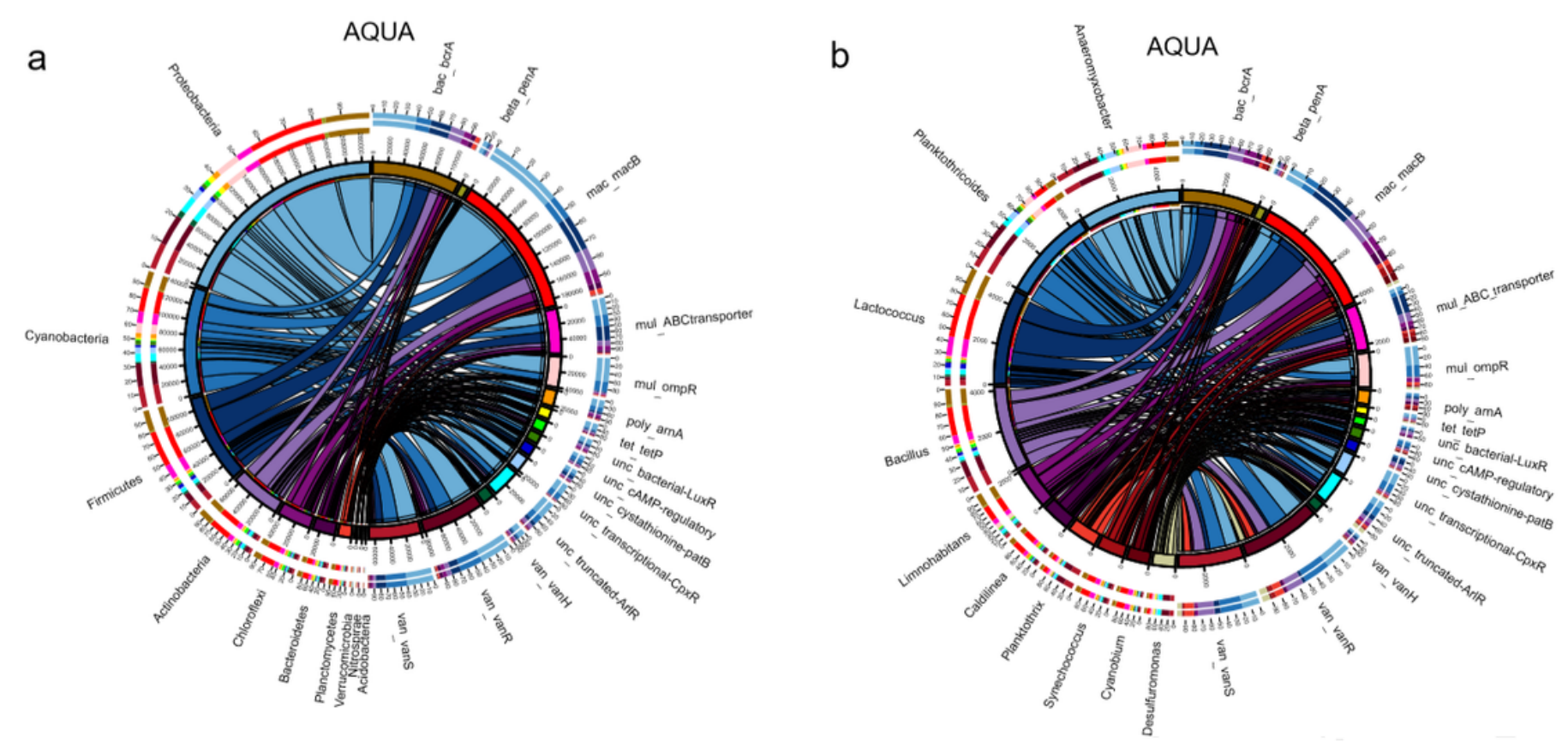

Figure 2

Circos analysis between the ARG subtypes and the microbiota carrying ARGs. a The top 15 most abundant ARG subtypes and the top ten most abundant phyla carrying ARGs. b The top 15 most abundant ARG subtypes and the top ten most abundant genera carrying ARGs. 


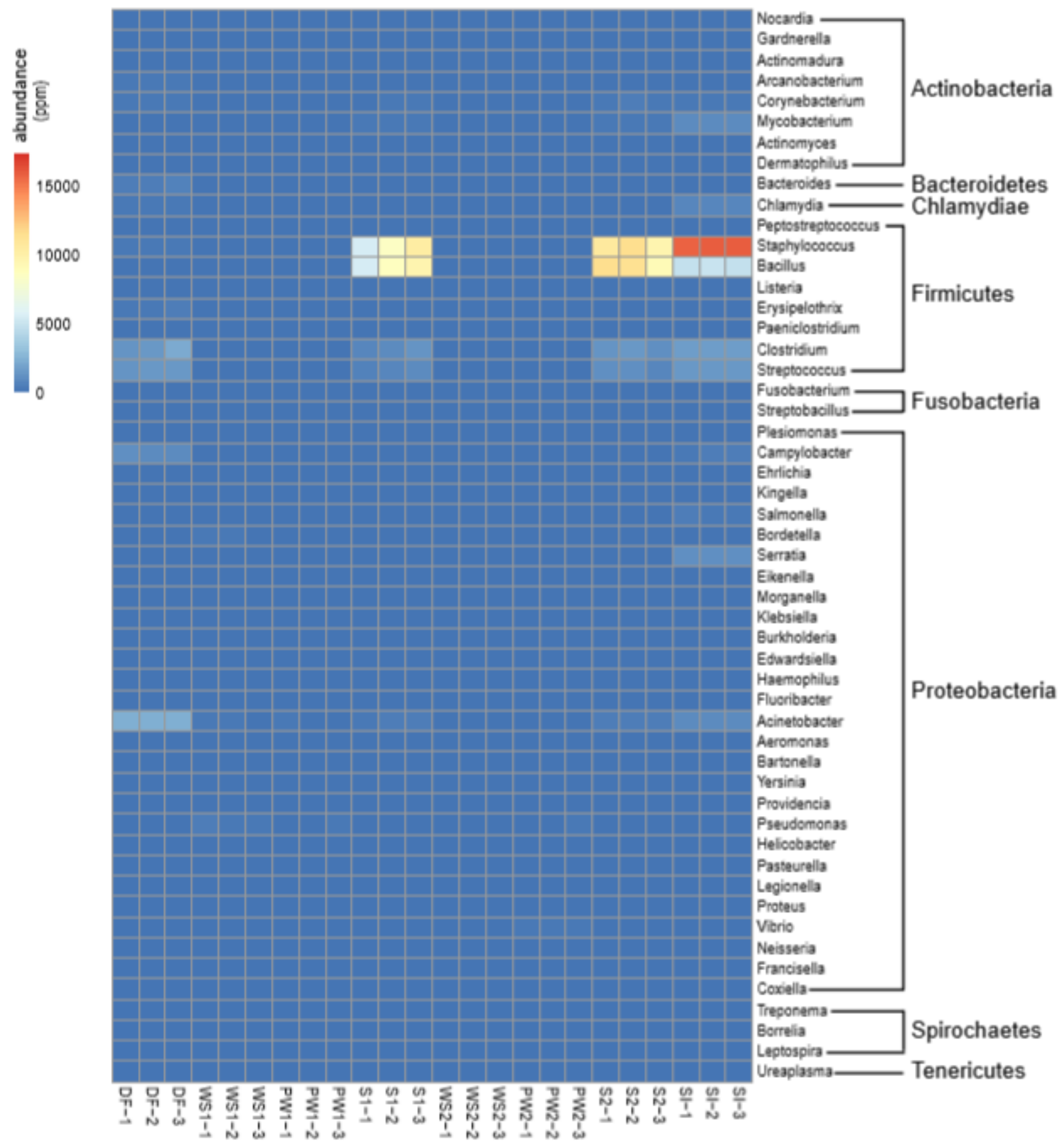

Figure 3

Abundances of human pathogenic bacteria (HPB) for the samples in aquaculture. 

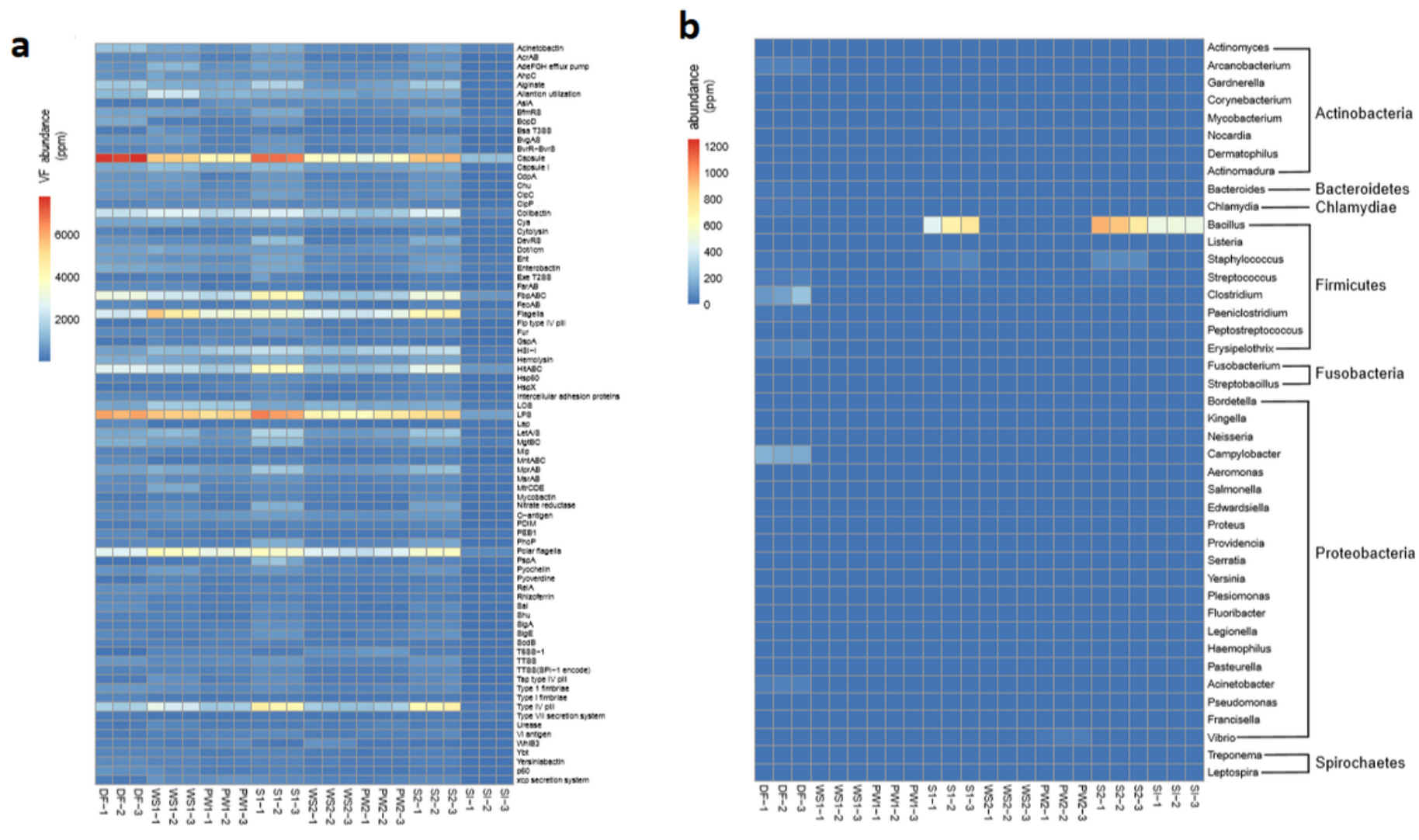

\section{Figure 4}

Abundances of virulence factors (VFs) and human pathogenic bacteria (HPB) carrying VFs for the samples in aquaculture. a Heatmap showing the abundances of VFs. b Heatmap showing the abundances of HPB carrying VFs for phyla and genera. 

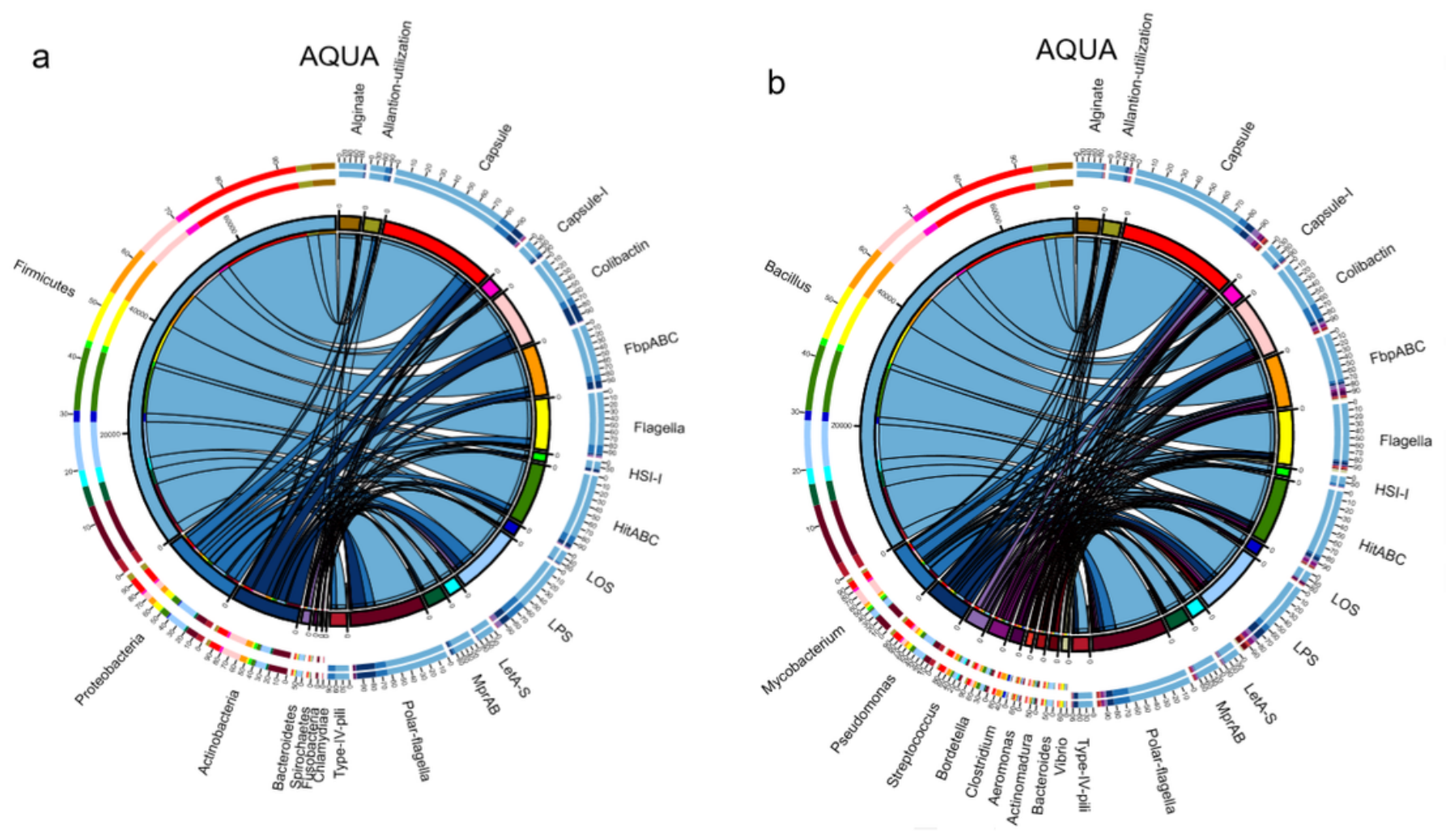

Figure 5

Circos analysis between the virulence factors (VFs) and the human pathogenic bacteria (HPB) carrying VFs. a The top 15 most abundant VFs and the top ten most abundant phyla carrying VFs. b The top 15 most abundant VFs and the top ten most abundant genera carrying VFs. 

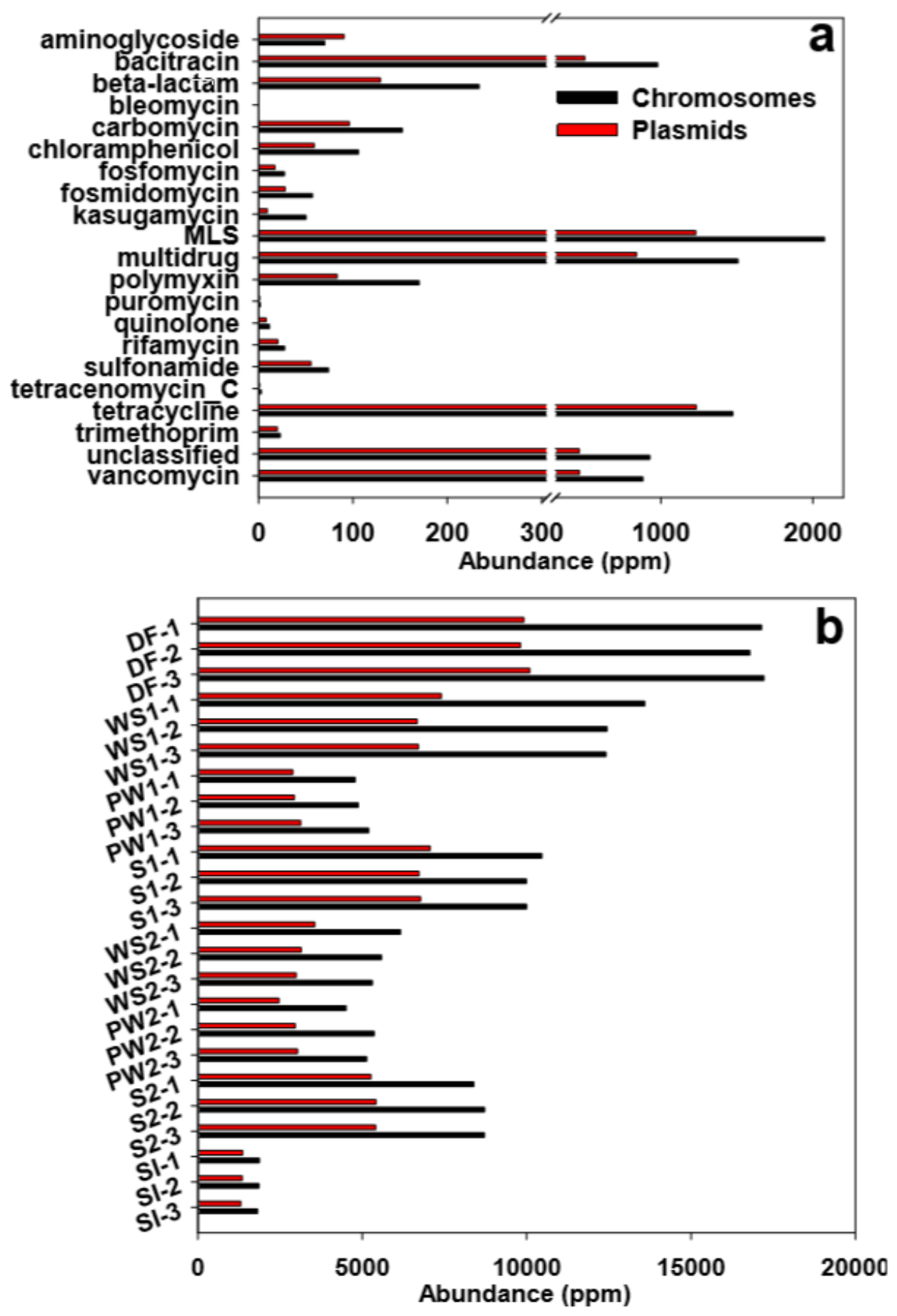

Figure 6

Abundances of ARGs types on different vectors. a Barplot showing the Abundances of ARGs types on bacterial chromosomes (black) or plasmids (red). b Barplot showing the total Abundance of ARGs types in the samples on bacterial chromosomes (black) or plasmids (red). 

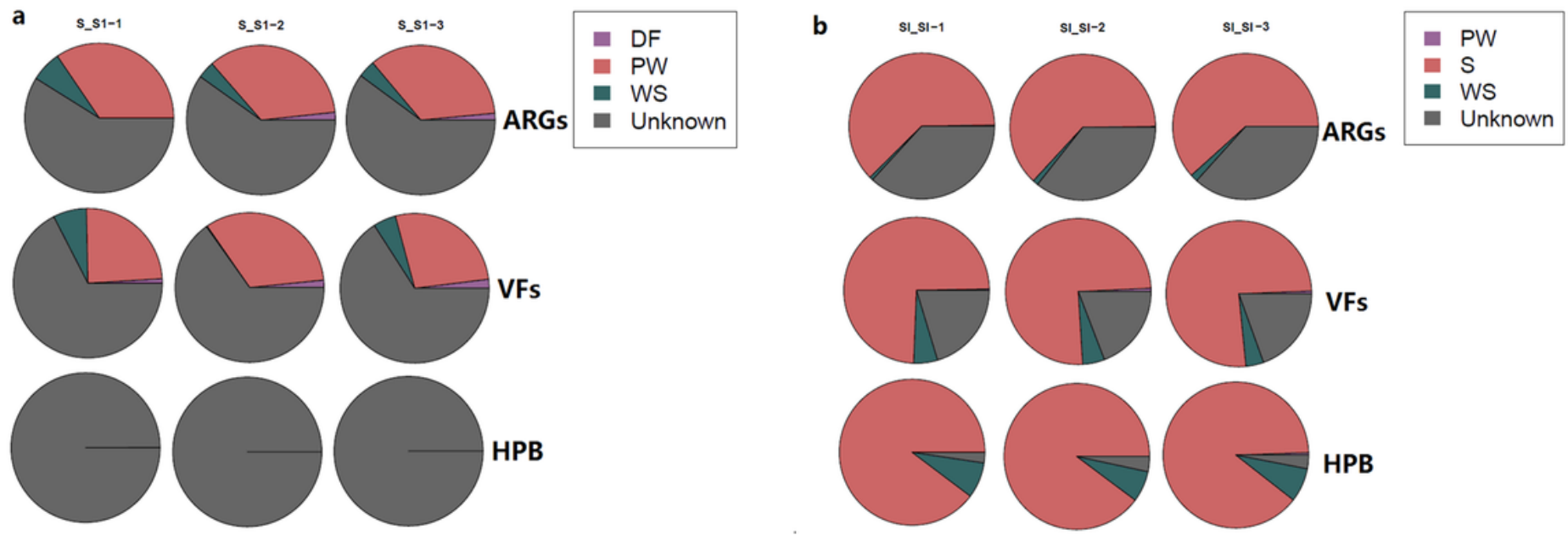

Figure 7

Source tracking analysis of microbiota in the samples. a Pieplot showing the sources of microbiota in the sediment for ARGs, VFs and HPB. b Pieplot showing the sources of microbiota in the shrimp gut samples for ARGs, VFs and HPB. DF, duck feces; PW, pond water; WS, water source; S, sediment; Unknow, an unknown source. 